Revue internationale P.M.E.

Économie et gestion de la petite et moyenne entreprise

Revue

internationale

PME

\title{
À la recherche du cœur de l'entrepreneuriat : vers une nouvelle vision du domaine
}

\section{Alain Fayolle}

Volume 17, numéro 1, 2004

URI : https://id.erudit.org/iderudit/1008453ar

DOI : https://doi.org/10.7202/1008453ar

Aller au sommaire du numéro

\section{Éditeur(s)}

Presses de l’Université du Québec

ISSN

0776-5436 (imprimé)

1918-9699 (numérique)

Découvrir la revue

Citer cette note

Fayolle, A. (2004). À la recherche du cœur de l'entrepreneuriat : vers une nouvelle vision du domaine. Revue internationale P.M.E., 17(1), 101-121. https://doi.org/10.7202/1008453ar

\section{Résumé de l'article}

Ce travail présente des réflexions relatives à la formulation d'une nouvelle vision de l'objet de recherche au cœur, selon nous, de la recherche en entrepreneuriat. Nous décrivons, notamment, le concept de «situation entrepreneuriale ». Par situation entrepreneuriale, nous entendons toute situation qui relie un individu, très fortement engagé (consommation de ressources vitales : temps, argent, énergie, etc.) dans une action entrepreneuriale (comportements, décisions, activités, etc.), et un projet ou une organisation utilisé comme support de création de valeur. Une des

caractéristiques, voire une des spécificités de la perspective que nous adoptons est que nous ne nous intéressons pas seulement à un acteur (individu ou groupe d'individus), une organisation ou une entreprise, mais bien davantage à un couple sujet-objet, individu-projet ou encore individu-organisation. $\mathrm{Ce}$ couple ne peut pas être dissocié dans toute approche scientifique (observation, analyse) d'une situation entrepreneuriale. Nous pensons donc qu'une situation entrepreneuriale peut être caractérisée par une conjonction sujet-objet et par un niveau de proximité ou de distance entre ces deux éléments. Dans ces conditions, une situation entrepreneuriale pourrait être qualifiée par la «mesure » de la distance séparant l'objet et le sujet impliqués dans cette situation. L'objet de recherche au cœur de l'entrepreneuriat correspond, de notre point de vue, à ces situations entrepreneu-riales qu'il est nécessaire de décrire et d'expliquer dans leurs dimensions statique et dynamique, afin que les connaissances produites par ce type de recherche puissent faciliter l'action des entrepreneurs et de ceux qui les accompagnent, tout en accroissant l'efficience des processus d'action et de décision.
Ce document est protégé par la loi sur le droit d'auteur. L'utilisation des services d'Érudit (y compris la reproduction) est assujettie à sa politique d'utilisation que vous pouvez consulter en ligne.

https://apropos.erudit.org/fr/usagers/politique-dutilisation/ 


\title{
À la recherche du cœur de l'entrepreneuriat: vers une nouvelle vision du domaine
}

Alain FAYOLLE

INPG-Esisar

CERAG, EPI

\section{MOTS CLÉS}

\section{Recherche en entrepreneuriat - Processus entrepreneurial Situation entrepreneuriale}

\begin{abstract}
RÉSUMÉ
Ce travail présente des réflexions relatives à la formulation d'une nouvelle vision de l'objet de recherche au cœur, selon nous, de la recherche en entrepreneuriat. Nous décrivons, notamment, le concept de "situation entrepreneuriale ". Par situation entrepreneuriale, nous entendons toute situation qui relie un individu, très fortement engagé (consommation de ressources vitales : temps, argent, énergie, etc.) dans une action entrepreneuriale (comportements, décisions, activités, etc.), et un projet ou une organisation utilisé comme support de création de valeur. Une des caractéristiques, voire une des spécificités de la perspective que nous adoptons est que nous ne nous intéressons pas seulement à un acteur (individu ou groupe d'individus), une organisation ou une entreprise, mais bien davantage à un couple sujet-objet, individu-projet ou encore individu-organisation. Ce couple ne peut pas être dissocié dans toute approche scientifique (observation, analyse) d'une situation entrepreneuriale. Nous pensons donc qu'une situation entrepreneuriale peut être caractérisée par une conjonction sujet-objet et par un niveau de proximité
\end{abstract}

\section{L'AUTEUR}

ALAIN FAYOLLE est maître de conférences à l'Institut national polytechnique de Grenoble après avoir été pendant 10 ans professeur d'entrepreneuriat et de management stratégique à l'EM Lyon. Il est également chercheur au CERAG et dirige une équipe de recherche technologique, EPI, de l'Université Pierre-Mendès-France et de l'Institut national polytechnique de Grenoble, spécialisée dans l'étude des processus de création d'activités innovantes. Adresse: INPGESISAR, 50, rue Barthélemy-de-Laffemas, B.P. 54, 26902 Valence, Cédex 9, France. Courriel : <alain.fayolle@esisar.inpg.fr>. 
ou de distance entre ces deux éléments. Dans ces conditions, une situation entrepreneuriale pourrait être qualifiée par la «mesure» de la distance séparant l'objet et le sujet impliqués dans cette situation. L'objet de recherche au cour de l'entrepreneuriat correspond, de notre point de vue, à ces situations entrepreneuriales qu'il est nécessaire de décrire et d'expliquer dans leurs dimensions statique et dynamique, afin que les connaissances produites par ce type de recherche puissent faciliter l'action des entrepreneurs et de ceux qui les accompagnent, tout en accroissant l'efficience des processus d'action et de décision.

\begin{abstract}
This work presents our thoughts on a new definition of the research object at the heart of the research in entrepreneurship. We therefore propose a conceptual model and a key concept which is the "entrepreneurial situation". An "entrepreneurial situation" is any situation which links a strongly committed individual (use of vital resources: time, money, energy, etc.) to an entrepreneurial action (decisions, behaviours, tasks, etc.) and a project or an organisation. One of the characteristics, or even specificity of our perspective is that we are not looking at just an actor, individual or group, an organisation or company, but rather a subject-object, or individual-project, individual-organisation couple. This couple can not be separated in any scientific approach (observation, analysis) of an "entrepreneurial situation". We therefore think that an "entrepreneurial situation" can be characterised by a subject-object conjunction, and by a level of proximity or distancing index between the two elements. Under such conditions, an "entrepreneurial situation" could be qualified by the "measuring" of a distance or proximity between subject and object involved in it. The object of research at the heart of entrepreneurship corresponds, we feel, to these "entrepreneurial situations" which need describing, explaining and including in their dynamism and evolution, so that from the scientific knowledge produced, the actions of entrepreneurs and accompanying support structures can be made easier and more efficient.
\end{abstract}

\title{
RESUMEN
}

Este trabajo presenta reflexiones relacionadas a la formulación de una nueva visión del objeto de investigación en el centro, según nosotros, de la investigación empresarial. Nosotros describimos, especialemente, el concepto de «situación empresarial ». Por situación empresarial, nosotros entendemos toda situación que asocia un individuo, fuertemente comprometido (consumo de recursos vitales: tiempo, dinero, energía...) en una acción empresarial (comportamientos, decisiones, actividades...), y un proyecto o una organización utilizada como soporte de creación de valor. Una de las características, incluso una de las especificidades de la perspectiva que nosotros adoptamos es que no nos interesamos solamente por un actor (individuo u grupo de individuos), una organización o una empresa, sino por una pareja sujeto/objeto, individuo/proyecto u individuo/organización. Este duo no puede estar disociado en todo enfoque científico (observación, analisis) de una situación empresarial. Nosotros pensamos, entonces, que una situación empresarial puede ser caracterizada por una conjunción sujeto / objeto y por un nivel de proximidad o de distancia entre estos dos elementos. En estas condiciones, una situación empresarial podria ser calificada por la «medida» de la distancia 
separando el objeto y el sujeto implicados en esta situación. El objeto de la investigación en el centro de la empresa corresponde, desde nuestro punto de vista, a esas situaciones empresariales donde es necesario describir y explicar en sus dimensiones estática y dinámica, de manera que los conocimientos producidos por este tipo de investigación puedan facilitar la acción de los empresarios y de aquellos que los acompañan, siempre aumentando la eficiencia de los procesos de acción y de decisión.

\section{ZUSAMMENFASSUNG}

Diese Arbeit stellt Überlegungen betreffend der Formulierung eines neuen Forschungsobjekts dar, der Forschung in Unternehmertum. Wir beschreiben insbesondere das Konzept der unternehmerischen Situation. Unter einer unternehmerischen Situation verstehen wir jede Situation, welche ein Individuum an eine unternehmerische Aktion (Verhalten, Entscheidungen, Aktivitäten usw.) und an ein werteschaffendes Projekt verpflichtet und verbindet. Eine der Eigenschaften und sogar eine der Besonderheiten der Perspektive, die wir annehmen, ist, dass wir uns nicht nur für einen Beteiligten (Individuum oder Gruppeindividuen), eine Organisation oder ein Unternehmen interessieren. Wir betrachten eine Verbindung zwischen Subjekt und Objekt, so beispielsweise Individuum/Projekt oder Individuum / Organisation. Wir denken, dass eine unternehmerische Situation charakterisiert ist durch diese Kombination und durch ein Nähe- oder ein Distanzniveau zwischen diesen zwei Elementen.

Der zentrale Forschungsgegenstand im Unternehmertum entspricht nach unserer Meinung der definierten Unternehmersituation. Diese Situation ist zu beschreiben und zu erklären in ihren statischen und dynamischen Dimensionen. Die Forschungserkenntnisse erlauben, einerseits das unternehmerische Verhalten zu erkennen und vereinfacht darzustellen um andererseits die Leistungsfähigkeit der Aktionsvorgänge und der Entscheidungen zu steigern.

\section{Introduction}

Le champ de l'entrepreneuriat est très diversifié et ses multiples composantes sont étudiées par des économistes, des sociologues, des historiens, des psychologues, des spécialistes des sciences du comportement et du management (Filion, 1997). Même si des contributions de recherche significatives ont été faites dans le domaine de l'entrepreneuriat, quelques chercheurs considèrent que l'étude scientifique de ce champ en est encore à ses premiers pas (Brazeal et Herbert, 1999). Les approches disciplinaires indépendantes ne suffisent plus aujourd'hui pour développer des connaissances de nature à expliquer un phénomène qui apparaît de plus en plus difficile à saisir (Wortman, 1987 ; Gartner, 1989 ; Bruyat et Julien, 2001). La complexité des objets et du champ de recherche accroît considérablement les problèmes qu'ont à résoudre les chercheurs et explique probablement en partie le manque de continuité dans les travaux de recherche (Wortman, 1987 ; Gartner, 1989 ; Bruyat, 1993).

Revue internationale P.M.E., vol. 17, nº 1, 2004 
Dans ces conditions, il n'est pas surprenant que nous constations depuis quelques années un très fort développement des contributions épistémologiques, théoriques et méthodologiques en entrepreneuriat. La plupart d'entre elles tendent à affirmer l'identité du champ et un positionnement en tant que champ scientifique autonome (Johannisson et Landström, 1999). De nouvelles questions de recherche apparaissent également qui tendent à souligner l'importance de quelques approches conceptuelles ${ }^{1}$ et révèlent la nécessité de se concentrer sur l'étude des processus (Bouchikhi, 1993 ; Bull et Willard, 1993 ; Venkataraman, 1997 ; Sarasvathy, 2000 ; Bruyat et Julien, 2001).

Nous inscrivons ce travail dans ce contexte de foisonnement d'idées et de travaux à la recherche, au fond, de perspectives nouvelles. L'objectif de notre contribution est de présenter une vision du domaine comprenant la définition d'un objet singulier de recherche et quelques idées sur la façon de l'étudier. La dernière section est entièrement consacrée à ce projet de représentation de ce que nous pensons être au cœur du domaine (section 4). Pour en arriver à la formulation de ces conceptions qui constituent l'apport principal de la contribution, nous avons souhaité suivre un cheminement particulier. Tout d'abord, nous avons voulu montrer, même brièvement, toute la diversité qui caractérise aujourd'hui le champ de l'entrepreneuriat et les questionnements de recherche (section 1). Pour bien insister sur le fait que notre regard n'est qu'une façon, parmi d'autres, de voir le domaine et que notre objet ne s'intéresse qu'à un sous-ensemble des questions qui se posent dans le champ. Nous avons ensuite resserré notre analyse de la littérature en la focalisant sur trois principaux courants de recherche qui s'intéressent à la notion de processus (section 2). Cela est important pour nous dans la mesure où nous inscrivons nos travaux en général et celui-ci, en particulier, dans cette orientation processus. Nous essayons d'exposer les raisons qui nous amènent à écarter les deux premières écoles et à ne retenir la troisième que d'une façon partielle. Il nous a semblé difficile, enfin, d'entrer dans la formulation d'une vision nouvelle du domaine sans expliciter notre positionnement de chercheur (section 3), lequel influence, nous semble t-il, la visée et la manière dont nous regardons l'objet.

\section{La diversité des domaines et des questions de recherche}

Le caractère multidisciplinaire du champ de l'entrepreneuriat a déjà été démontré (Bruyat, 1993 ; Filion, 1997 ; Tornikoski, 1999). Dans ces conditions, il apparaît presque illusoire de donner une représentation complète et détaillée de la couver-

1. Ces approches sont construites principalement autour des concepts d'opportunité et d'organisation émergente. 
ture de ce champ par toutes les différentes disciplines qui s'y sont intéressées. Cela étant, nous avons souhaité, dans un but pédagogique, construire un tableau de synthèse présentant les principales disciplines et leur articulation dans des problématiques de recherche (voir tableau 1). Le tableau proposé donne les principales orientations et caractéristiques méthodologiques pour chacune des principales questions de recherche en entrepreneuriat : «quoi», « qui et pourquoi », « comment». Sa lecture doit être faite avec beaucoup de prudence, car, dans ce travail, seuls les principaux courants sont mentionnés. Cela signifie que, dans certains cas, des informations peuvent être absentes. Par exemple, des sociologues, comme Aldrich (1999) se sont intéressés à la question du «comment» et parfois, des approches quantitatives ont été ou sont utilisées pour étudier le processus entrepreneurial.

\section{TABLEAU 1}

\section{Vue organisée et synthétique des recherches en entrepreneuriat*}

\begin{tabular}{|c|c|c|c|}
\hline Question principale & Quoi & Qui / Pourquoi & Comment \\
\hline Types d'approches & $\begin{array}{l}\text { Approches } \\
\text { fonctionnelles }\end{array}$ & $\begin{array}{l}\text { Approches } \\
\text { sur les individus }\end{array}$ & $\begin{array}{l}\text { Approches } \\
\text { sur les processus }\end{array}$ \\
\hline Échelle du temps & 200 dernières années & $\begin{array}{l}\text { Depuis le début des } \\
\text { années } 1950 .\end{array}$ & $\begin{array}{l}\text { Depuis le début des } \\
\text { années } 1990 .\end{array}$ \\
\hline $\begin{array}{l}\text { Domaine } \\
\text { scientifique } \\
\text { principal }\end{array}$ & Économie & $\begin{array}{l}\text { Psychologie, sociologie, } \\
\text { psychologie cognitive, } \\
\text { anthropologie sociale. }\end{array}$ & $\begin{array}{l}\text { Sciences de gestion } \\
\text { Sciences de l'action } \\
\text { Théories des organisations }\end{array}$ \\
\hline Objet d'étude & $\begin{array}{l}\text { Fonctions } \\
\text { de l'entrepreneur }\end{array}$ & $\begin{array}{l}\text { Caractéristiques } \\
\text { personnelles. } \\
\text { Traits des individus } \\
\text { entrepreneurs et } \\
\text { entrepreneurs potentiels. }\end{array}$ & $\begin{array}{l}\text { Processus de création: } \\
\text { - d'une nouvelle activité, } \\
\text {-d'une nouvelle } \\
\text { entreprise. }\end{array}$ \\
\hline $\begin{array}{l}\text { Paradigme } \\
\text { dominant }\end{array}$ & Positivisme & $\begin{array}{l}\text { Positivisme } \\
\text { Sociologie compréhensive }\end{array}$ & Constructivisme \\
\hline Méthodologie & Quantitative & $\begin{array}{l}\text { Quantitative } \\
\text { Qualitative }\end{array}$ & Qualitative \\
\hline Hypothèse de base & $\begin{array}{l}\text { L'entrepreneur joue / } \\
\text { ne joue pas un rôle } \\
\text { important dans } \\
\text { la croissance économique. }\end{array}$ & $\begin{array}{l}\text { Les entrepreneurs sont } \\
\text { différents des non- } \\
\text { entrepreneurs. }\end{array}$ & $\begin{array}{l}\text { Les processus } \\
\text { entrepreneuriaux sont } \\
\text { différents les uns } \\
\text { des autres. }\end{array}$ \\
\hline $\begin{array}{l}\text { Lien avec la } \\
\text { demande sociale } \\
\text { (qui est } \\
\text { intéressé par...) }\end{array}$ & $\begin{array}{l}\text { État, collectivités } \\
\text { territoriales, } \\
\text { responsables } \\
\text { économiques. }\end{array}$ & $\begin{array}{l}\text { Entrepreneurs } \\
\text { Entrepreneurs potentiels } \\
\text { Système éducatif } \\
\text { Formateurs }\end{array}$ & $\begin{array}{l}\text { Entreprises } \\
\text { Entrepreneurs } \\
\text { Entrepreneurs potentiels } \\
\text { Éducateurs et formateurs } \\
\text { Structures } \\
\text { d'accompagnement et } \\
\text { d'appui des entrepreneurs. }\end{array}$ \\
\hline
\end{tabular}

\footnotetext{
* Ce tableau a été élaboré à partir d'éléments proposés par Landström (1998), Filion (1997) et Tornikoski (1999).
} 
Les bases historiques de l'entrepreneuriat viennent des sciences économiques. Les approches fonctionnelles des économistes s'intéressent prioritairement aux effets de l'entrepreneuriat et au rôle de l'entrepreneur dans le développement du système économique.

Les approches centrées sur les individus visent à produire des connaissances sur les caractéristiques psychologiques des entrepreneurs, leurs traits de personnalité, leurs motivations, leurs comportements, leurs origines et trajectoires sociales. Elles cherchent peut-être aussi un profil type d'entrepreneur qu'il serait possible de reconnaître par une caractéristique principale ou un ensemble de caractéristiques. Une des premières questions relatives aux individus a porté (et porte encore) sur le caractère inné de l'entrepreneur. Les entrepreneurs naissent-ils avec un sixième sens, une sorte d'instinct entrepreneurial ? Certains ne sont pas loin de le penser ${ }^{2}$. Mais, beaucoup d'autres, chercheurs et praticiens, réfutent cette hypothèse. Les spécialistes des sciences du comportement humain ont donc multiplié les recherches pour tenter d'analyser et de comprendre les traits, les types et les comportements de l'entrepreneur.

L'apparition récente des approches sur les processus est justifiée par l'idée, de plus en plus admise, d'une très grande diversité dans les situations entrepreneuriales et dans les créations d'entreprises (Bruyat, 1993, 1994). Comme le souligne Gartner (1985), il existe des différences importantes entre les entrepreneurs, à tel point que, parfois, la dispersion est plus faible lorsque l'on compare des entrepreneurs à des non-entrepreneurs, que lorsque l'on compare des entrepreneurs entre eux. Les questions auxquelles s'interesse ce courant de recherche partent également du principe que l'entrepreneuriat est considéré comme un phénomène complexe et multidimensionnel (Gartner, 1985 ; Cunningham et Lischeron, 1991 ; Bruyat et Julien, 2001).

Pour conclure cette section, ce qui nous semble devoir caractériser l'évolution des recherches en entrepreneuriat au cours des dernières années est, d'une part, la réorientation du centre focal qui s'est déplacé de l'individu vers le processus (Bygrave et Hofer, 1991) et, d' autre part, l'abandon progressif d'une vision statique pour une vision plus dynamique. Il nous semble enfin que les recherches s'ancrent davantage dans des épistémologies constructivistes et utilisent des approches plus qualitatives, comme l'attestent les thèses soutenues dans le champ de l'entrepreneuriat, en France, au cours des années $1990^{3}$.

2. Voir les auteurs cités par Cunnigham et Lischeron (1991), dans le paragraphe consacré à «The Great Person School of Entrepreneurship ».

3. Thèses de Bruyat (1993), Sammut (1995), Fayolle (1996), De la Ville (1996), Verstratete (1997) et Marion (1999). 


\section{Le processus au cœur du domaine de recherche}

Parmi les travaux les plus prometteurs qui placent le processus entrepreneurial au cœur de leurs préoccupations, trois approches semblent aujourd'hui prendre de l'importance. Elles décrivent des conceptions assez différentes de l'entrepreneuriat en tant que domaine de recherche. Le premier courant impulsé par Gartner (1988, 1990, 1993), qui reste un auteur très représentatif de cette façon de penser l'objet de recherche, défend l'idée que l'entrepreneuriat est la création de nouvelles organisations. Dans cette vision, l'étude de l'entrepreneuriat revient à étudier la naissance de nouvelles organisations, c'est-à-dire les activités permettant à un individu de créer une nouvelle entité. L'émergence organisationnelle est donc le processus qui conduit à l'apparition d'une nouvelle organisation. La conception de Gartner a été adoptée par d'autres auteurs (Aldrich, 1999; Thornton, 1999; Sharma et Chrisman, 1999; Hernandez, 2001), ce qui a permis la constitution d'une communauté de chercheurs qui consacre l'essentiel de ses travaux à l'étude du phénomène d'émergence organisationnelle. On pourrait également rattacher à ce courant des chercheurs comme Bouchiki (1993) et Verstraete (1999). Cette approche présente, de notre point de vue, un inconvénient majeur. Les chercheurs qui s'inscrivent dans ce courant s'intéressent tout autant, sinon plus, à la création d'organisation, sous-ensemble de la théorie des organisations, qu'à l'entrepreneuriat (Shane et Venkataraman, 2000 ; Davidsson, Low et Wright, 2001 ; Wiklund, Dahlqvist et Havnes, 2001). En fonction du mode d'exploitation retenu pour valoriser une opportunité ou une invention, création d'une entreprise ou utilisation d'une organisation existante, le processus en question est entrepreneurial ou ne l'est pas. Par ailleurs, comme le montre Bruyat (1993), toutes les créations d'organisation ne conduisent pas à des situations où l'intensité du changement pour l'individu et l'importance de la création de valeur se situent à un niveau élevé. Des entreprises peuvent être créées par imitation, par reproduction ou encore dans le but de transférer une activité existante.

La deuxième conception, basée sur la notion d'opportunité entrepreneuriale, est celle de Shane et Venkataraman (2000), qui s'inscrit dans un courant de recherche déjà ancien (Stevenson et Jarillo, 1990; Bygrave et Hofer, 1991 ; Venkataraman, 1997). Le champ de l'entrepreneuriat est défini, ici, comme «L'étude académique de comment, par qui et avec quels effets, les opportunités qui conduisent à la création de futurs biens et services sont découvertes, évaluées et exploitées » (Venkataraman, 1997). Dans ces conditions, les processus de découverte, d'évaluation et d'exploitation des occasions d'affaires représentent des objets d'étude et de recherche essentiels. Des travaux récents ${ }^{4}$ (Wicklund, Dahlqvist et Havnes,

4. Voir également les actes des deux dernières conférences de Babson (Frontiers of Entrepreneurship Research) qui montrent que de très nombreux travaux s'inscrivent maintenant dans ce courant.

Revue internationale P.M.E., vol. 17, nº 1, 2004 
2001 ; Van der Veen et Wakkee, 2002) s'inscrivant dans ce courant centré sur les occasions d'affaires explorent conceptuellement ou empiriquement un aspect particulier du domaine ainsi défini. Cette perspective, comme la précédente, porte sur l'émergence, mais il s'agit ici de l'émergence d'une nouvelle activité économique qui n'est pas nécessairement liée à la création d'une nouvelle organisation. Cette approche présente également des inconvénients majeurs eu égard à l'idée que nous avons de l'étude du processus entrepreneurial. Elle présuppose, tout d'abord, que les occasions d'affaires existent dans la nature en tant que telles et qu'il suffit d'avoir une capacité à les reconnaître pour se les approprier et les transformer en réalités économiques : «Même si la reconnaissance des opportunités entrepreneuriales relève d'un processus subjectif, les opportunités, elles-mêmes sont des entités objectives qui ne sont pas perçues par tout le monde au même moment » (Shane et Venkataraman, 2000, p. 220). Nous pensons, pour notre part, que la possibilité entrepreneuriale se construit au cours du processus de création de l'activité et non pas qu'elle est le point de départ, élément «objectif» qu'il faut découvrir, de ce processus. Le second problème que nous relevons dans cette approche est lié au fait qu'elle préconise de se focaliser sur un processus d'exploitation (ou de concrétisation) d'une occasion d'affaires qui va déboucher sur la création d'un produit ou d'un service. Il nous semble que l'entrepreneuriat pourrait également tirer bénéfice de l'étude de processus n'ayant pas abouti, dès lors que l'objectif est de mieux comprendre ce qui se passe dans ces situations entrepreneuriales. Quelques chercheurs suédois essaient d'ailleurs de développer des projets et des travaux de recherche qui, tout en s'inscrivant dans ce deuxième courant de pensée, s'efforcent d'en atténuer les limites ci-dessus mentionnées.

La troisième conception est, entre autres, celle de Bruyat (1993). Pour cet auteur, «l'objet scientifique étudié dans le champ de l'entrepreneuriat est la dialogique individu / création de valeur » (Bruyat, 1993, p. 57). Le principe dialogique proposé par Morin (1989) signifie que deux ou plusieurs logiques sont liées en une unité, de façon complexe (complémentaire, concurrente et antagoniste) sans que la dualité se perde dans l'unité. Cette dialogique s'inscrit dans une dynamique de changement et peut être définie comme suit (Bruyat, 1993, p. 58) :

«[...] l'individu est une condition nécessaire pour la création de valeur, il en détermine les modalités de production, l'ampleur... Il en est l'acteur principal. Le support de la création de valeur, une entreprise par exemple, est la «chose » de l'individu, nous avons :

\section{INDIVIDU $\rightarrow$ CRÉATION DE VALEUR}

La création de valeur, par l'intermédiaire de son support, investit l'individu qui se définit, pour une large part, par rapport à lui. Elle occupe une place prépondérante dans sa vie (son activité, ses buts, ses moyens, son statut social, etc.), elle est susceptible de modifier ses caractéristiques (savoir-faire, valeurs, attitudes, etc.), nous avons :

\section{CRÉATION DE VALEUR $\rightarrow$ INDIVIDU}


Le système entrepreneurial (création de valeur $\leftrightarrow \rightarrow$ individu) est en interaction avec son environnement et se trouve «embarqué » dans un processus par rapport auquel le temps constitue une dimension incontournable (Bruyat et Julien, 2001).

Cette représentation est, au fond, proche de celle de Gartner (1985) dans laquelle les dimensions importantes sont : l'individu ou les individus, l'environnement, l'organisation et le processus ${ }^{5}$. Le système défini par Bruyat est un système de type 9 au sens que lui donnent Boulding (1956) et Le Moigne (1990). Il est capable d'apprendre et de créer; il est également doté d'intention. Il s'inscrit dans un processus et une dynamique durant lesquels il est susceptible de se transformer. De nombreux travaux (Vesper, 1989; Woo et al., 1990) ont montré que les stratégies ou même les projets peuvent changer de manière significative même dans les tout premiers temps du processus. De plus, ce système est un système ouvert. Il interagit avec son environnement, il le subit, il en tire des ressources et des occasions favorables. Dans une certaine mesure, il peut le choisir et l'aménager (Marchesnay et Julien, 1989). Il peut aussi être stimulé par lui ou par les réseaux ou les milieux avec lesquels il interagit (Johannisson, Karlsson et Westin, 1994 ; Conti, Malecki et Oinas, 1995).

Notre vision s'inspire beaucoup de l'approche de Bruyat. En particulier, nous pensons comme lui que le couple individu-projet ne peut être dissocié dans la définition de l'objet d'étude, compte tenu des relations qui les unissent. Cette approche pose quelques problèmes, néanmoins, dans une perspective dynamique dans les réponses qu'elle n'apporte pas ou peu à des questions importantes, liées au moment où l'on entre dans une «situation entrepreneuriale» et au moment où l'on en sort. Pour nous, ces questions sont essentielles et sont au cœur du phénomène et du domaine.

\section{Quel positionnement pour le chercheur?}

Essayer de définir le cœur de l'entrepreneuriat n'est pas seulement une affaire de définition des frontières du champ de recherche ; c'est aussi et surtout une question d'organisation des connaissances et de positionnement des chercheurs par rapport au domaine scientifique. À cet égard, «en tant que système social concret d'action, le champ de recherche en entrepreneurship est l'espace social et collectif où se déploient les stratégies des chercheurs et le système de leur positions relatives »

5. Si ce n'est que le processus n'est pas conceptualisé de la même façon par ces deux auteurs. Dans les premiers travaux de Gartner (1985), le processus est assimilé à une manière de faire, à une stratégie. 
(Dery et Toulouse, 1994, p. 4 et 5). Avant d'entrer dans notre conception de ce qu'est le cœur de la recherche en entrepreneuriat, il apparaît utile d'avancer quelques réflexions sur le rôle et la place du chercheur en entrepreneuriat.

Bien évidemment, ces éléments ne peuvent être dissociés d'une posture épistémologique construite dans la durée et que nos activités de recherche dans le champ ont contribué à faire émerger et à stabiliser ${ }^{6}$.

Le chercheur en entrepreneuriat appartient, tout d'abord, à une communauté scientifique d'origine. Dans notre cas, il s'agit des sciences de gestion. Dans ce contexte, la recherche en entrepreneuriat peut être vue comme de nature à permettre une meilleure compréhension de l'action des entrepreneurs, pour les entrepreneurs eux-mêmes, afin de les aider à mieux réaliser ce qu'ils font ou doivent faire, et pour ceux qui les accompagnent, les conseillent, les aident et les forment, pour qu'ils améliorent l'efficience de leurs approches. Ce premier préalable a pour objectif de ne pas perdre de vue la finalité que devrait avoir tout projet ou programme de recherche en entrepreneuriat. Décrire, expliquer, comprendre, prédire, changer ${ }^{7}$ l'action des entrepreneurs doit pouvoir s'envisager, nous semble-t-il, à travers un couplage étroit avec des terrains entrepreneuriaux. Or, beaucoup de recherches en gestion et en entrepreneuriat sont faites aujourd'hui avec, pour le moins, beaucoup de distance par rapport au terrain et une insuffisance de données empiriques. À tel point qu'on peut se demander légitimement si le terrain est légitime ${ }^{8}$.

D'autre part, il semblerait que dans certaines disciplines des sciences de gestion un fossé soit en train de se creuser entre les chercheurs et les praticiens (Paturel et Savall, 1999). Les seconds ne comprennent pas les premiers parce que les sujets traités sont éloignés de leurs préoccupations et que le langage des scientifiques ne leur est pas accessible. Ce constat pourrait être également fait dans le champ de l'entrepreneuriat.

Le second préalable est donc qu'il convient, dans la mesure du possible, de rapprocher la recherche en entrepreneuriat de la demande sociale et du terrain. Outre les entrepreneurs, les entrepreneurs potentiels et les professions et métiers concernés par l'entrepreneuriat, de nombreux autres acteurs économiques et politiques participent de cette demande sociale. Nous pensons à l'État, aux collectivités territoriales, aux organismes consulaires et aux entreprises. Pour illustrer notre

6. Voir sur le sujet de la construction d'une posture épistémologique, Baumard (1997).

7. Nous reprenons ici les objectifs ou catégories d'objectifs cités habituellement dans l'univers de la recherche en gestion. Voir principalement Wacheux (1996).

8. Titre d'un colloque scientifique organisé à Lyon le 8 décembre 1999 par le Pôle lyonnais de recherche en gestion comprenant les équipes investies dans le champ des universités Lyon II et Lyon III et de l'EM Lyon. 
propos, nous voudrions rapidement évoquer les demandes récentes du ministère de l'Éducation nationale, de la Recherche et de la Technologie concernant l'état actuel de l'enseignement de l'entrepreneuriat (Fayolle, 1999, 2000) et la création d'entreprises par des chercheurs. L'action de ce ministère en faveur de l'innovation, des incubateurs et de la valorisation de la recherche universitaire entraîne, de facto, l'émergence de nouvelles questions et une évolution de la demande sociale.

La position du chercheur dans un champ scientifique ainsi que l'idée qu'il se fait de son rôle social et la stratégie personnelle qu'il adopte constituent des éléments majeurs de nature à influencer fortement les objets, les finalités et les processus de ses recherches.

Dans le domaine qui nous intéresse, en réfutant l'idée d'une approche trop normative, les recherches réalisées par des personnes spécialisées en sciences de gestion devraient privilégier l'action des entrepreneurs observée dans des situations concrètes et scientifiquement définies, dans un souci de production de connaissances en phase avec la demande sociale.

\section{Au cœur de la recherche en entrepreneuriat}

Notre approche du cœur de l'entrepreneuriat part d'un double cheminement concernant les deux éléments clés du système décrit par Bruyat (1993) : l'individu et la création de valeur.

Le premier cheminement s'intéresse à l'acteur. Tout individu est, d'une certaine façon, un entrepreneur en puissance. Au regard de l'entrepreneuriat, un individu peut passer par différents stades dans ses parcours de vie personnelle et / ou professionnelle. Le premier stade, dans lequel il peut rester toute sa vie, est caractérisé par une indifférence à (ou une inconscience de) l'entrepreneuriat. Tout être humain peut, aussi, sous l'effet de nombreuses influences, développer une propension entrepreneuriale que nous définissons comme une sorte d'inclination, un penchant à s'engager, un jour, dans une démarche entrepreneuriale. On peut considérer qu'un individu manifestant une propension entrepreneuriale est un individu éveillé ou sensibilisé à l'entrepreneuriat (Fayolle, 1996). La propension peut évoluer vers l'intention entrepreneuriale qui signifie un dessein délibéré, une volonté d'accomplir un acte ou de développer un comportement entrepreneurial. Depuis quelques années, la théorie du comportement planifié (theory of planned behaviour) est mobilisée pour modéliser l'intention entrepreneuriale (Krueger et Carsrud, 1993 ; Kolvereid, 1996 ; Autio et al., 1997 ; Tkachev et Kolvereid, 1999). Cette théorie, ainsi que la plupart de celles utilisées dans cette première perspective, vient des champs de la sociologie et de la psychologie. L'intention précède assez souvent, mais pas toujours, le comportement entrepreneurial qui signifie à 
la fois une prise de décision de faire et un engagement dans l'action entrepreneuriale. Le stade ultime est celui de l'évaluation (avantages et bénéfices sur un plan individuel) des résultats de l'action entrepreneuriale.

Dans ce cheminement, chaque individu peut acquérir et développer un potentiel entrepreneurial défini comme un ensemble de ressources personnelles (motivations, connaissances, expériences, compétences, relations, aptitudes) utiles pour l'action entrepreneuriale.

Dans ce contexte, centré sur l'individu, les principales questions de recherche peuvent s'exprimer ainsi :

- Comment détecter et développer la propension entrepreneuriale des individus, en général, ou de telle catégorie d'individus, en particulier?

- Comment développer l'intention entrepreneuriale des individus, en général, ou de telle catégorie d'individus, en particulier?

- Comment faire pour obtenir un taux de transformation élevé entre l'intention et l'action entrepreneuriale, autrement dit, comment faciliter la prise de décision entrepreneuriale?

- Comment développer le potentiel entrepreneurial des individus, en général, ou de telle catégorie d'individus, en particulier? Comment le mesurer?

Le second cheminement concerne la création de valeur ou plutôt le support immatériel et / ou matériel de la création de valeur (son enveloppe ou ses enveloppes successives) et les concepts qui sont mobilisés pour le décrire. Ce support contient plus ou moins un potentiel de création de valeur, au sens large du terme, susceptible d'être libéré et de s'exprimer sous certaines conditions. Comme pour le premier cheminement, différents stades peuvent être repérés.

Le premier stade est l'idée entrepreneuriale qui peut jaillir dans la tête de n'importe quel individu, qui peut être aussitôt enfouie dans sa mémoire et oubliée définitivement ou qui peut être transformée en opportunité entrepreneuriale après un travail de confrontation avec l'environnement concerné et de première validation à travers un processus d'évaluation. À partir de l'occasion d'affaires, le projet entrepreneurial peut prendre forme, se structurer et faire l'objet, éventuellement, de formalisations matérielles. Le seul fait d'utiliser les concepts d'occasion d'affaires et de projet implique des interactions et des transactions avec des environnements et des milieux personnels et/ou professionnels. Le stade suivant correspond à l'émergence de l'organisation entrepreneuriale. Imaginée, visualisée, formalisée, dimensionnée dans un projet, elle va s'animer dès le lancement des 
activités et le moment du démarrage effectif. Le dernier stade est celui d'une organisation entrepreneuriale «stabilisée ${ }^{9}$ » qui peut être évaluée par l'intermédiaire d'indicateurs d'activités, de performances et de résultats.

Dans ce second cheminement, chaque stade renferme un potentiel de création de valeur plus ou moins important qui dépend de la qualité des observations, de celle du traitement des informations et de la pertinence des choix effectués. Néanmoins, la création effective de valeur n'intervient généralement qu'au cours du dernier stade. Cela pose la question de l'exploitation concrète de ce potentiel à travers un dispositif organisationnel, un niveau de ressources et des orientations et décisions stratégiques.

Mais le passage d'un stade à un autre n'est en rien automatique. Le cheminement peut s'interrompre pour différentes raisons (abandon d'une idée, d'une occasion d'affaires, d'un projet; disparition prématurée, volontaire ou non, d'une activité ou d'une entreprise). Le cheminement peut aussi se poursuivre sans heurts ou à travers des réorientations significatives.

Dans ce contexte, centré sur les supports de la création de valeur, les chercheurs peuvent vouloir répondre à des questions du type :

- Comment émergent les idées entrepreneuriales et comment développer la créativité entrepreneuriale?

- Comment reconnaître les opportunités entrepreneuriales ? D'où viennentelles ? Peut-on les construire ? Peuvent-elles être dissociées des individus qui les ont repérées?

- Comment les projets entrepreneuriaux se forment-ils et évoluent-ils?

- Comment les organisations sont-elles conçues et mises en place?

- Quels sont les facteurs clés de réussite d'une organisation entrepreneuriale?

Après ce double cheminement où chaque perspective a été analysée en grande partie indépendamment de l'autre, notre approche du cœur de l'entrepreneuriat va les réunir de façon à retrouver dans une même unité d'observation et d'analyse le

9. L'expression «stabilisée » indique simplement que l'organisation a atteint un niveau de fonctionnement où apparaissent des régularités et des repères. L'organisation peut toujours être, à ce stade de développement, dans une dynamique de changement importante liée principalement à la croissance des activités et à la nature des projets qui alimentent cette croissance. D'une certaine façon, l'organisation entrepreneuriale «stabilisée » voudrait marquer la fin du stade précédent. Le problème est qu'aujourd'hui il est difficile de savoir où, quand et comment se termine un processus d'émergence organisationnelle. 
couple individu-support de création de valeur. Pour nous, le cœur de l'entrepreneuriat et ce qui permet de définir l'objet scientifique est caractérisé par l'existence d'une relation forte entre les deux logiques en œuvre, celle du sujet et celle de l'objet.

Nous appelons «situation entrepreneuriale» toute situation reliant d'une façon très étroite, quasi indissociable, un individu caractérisé par un engagement personnel fort (consommation de ressources vitales : temps, argent, énergie) dans une action entrepreneuriale (décisions, comportements, tâches, etc.) et un projet ou une organisation émergente ou une organisation «stabilisée » de type entrepreneurial. Une des particularités, peut-être une spécificité du champ de l'entrepreneuriat, est que nous n'étudions pas uniquement un acteur, un individu ou un groupe, nous n'étudions pas non plus exclusivement une organisation ou une entreprise, mais nous étudions un couple sujet-objet, homme-projet, hommeorganisation. Ce couple ne peut pas être dissocié dans l'approche (observation et analyse) d'une situation entrepreneuriale. Cela nous amène à considérer qu'une situation entrepreneuriale peut être caractérisée par une conjonction sujet-objet et donc par un degré de proximité ou un indice de distanciation entre ces deux éléments. La perspective de recherche centrée sur la conjonction sujet-objet nous conduit à de nouvelles questions de recherche, telles celles-ci : Les phénomènes de conjonction et de disjonction sujet-objet résultent-ils de changement de phases ou d'état au cours du processus entrepreneurial ? La disjonction sujet-objet résultet-elle d'une désynchronisation des cycles de vie du projet (ou de l'organisation) et de l'entrepreneur? Comment conserver une trace de tous les événements reliés aux phénomènes de conjonction et disjonction sujet-objet? Une situation entrepreneuriale pourrait, dans ces conditions, être qualifiée par la «mesure » d'une distance ou proximité entre sujet et objet, comme l'indique la figure 1.

FIGURE 1

Une représentation du concept de situation entrepreneuriale

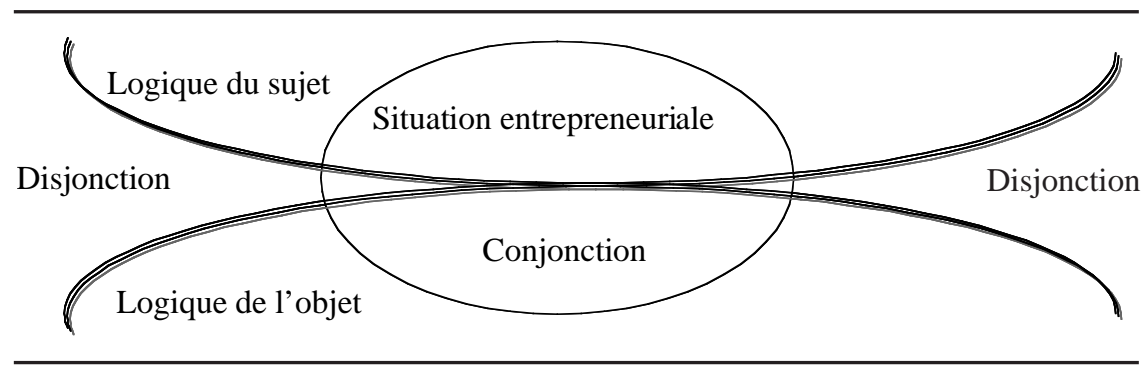

Dans une situation entrepreneuriale, les principaux comportements des individus sont liés à la prise d'initiatives, au changement souhaité et impulsé, à l'évaluation et à l'acceptation des risques et de leurs implications. Ils comprennent 
également l'ensemble des capacités utiles à l'identification, à l'appropriation et à la mise en œuvre des ressources nécessaires à l'action et à la concrétisation du projet, à l'émergence de l'organisation et à son fonctionnement.

Dans une situation entrepreneuriale, le support de la création de valeur peut être défini par un projet de création d'une activité ou d'une entreprise, le développement d'un produit ou d'une innovation, l'acquisition d'une entreprise ou d'une activité. Ce projet s'insère dans un environnement organisationnel, social, culturel, économique plus ou moins favorable. Une des caractéristiques clés de la situation considérée est l'existence et la perception d'un degré d'incertitude élevé (lié à l'intensité du changement pour l'individu et pour l'environnement) et l'importance des enjeux individuels et / ou collectifs qui placent souvent à un haut niveau de conscience les perceptions des risques encourus.

Le temps joue un rôle essentiel, car il est facteur d'évolution et de transformation du projet et de l'organisation de type entrepreneurial. Une situation peut être entrepreneuriale à un moment donné et ne plus l'être peu de temps après. Certains comportements individuels peuvent s'atténuer, perdre de leur intensité et parfois même disparaître pour laisser place à d'autres attitudes moins entrepreneuriales. Les contextes d'action évoluent comme les perceptions que les hommes s'en font. C'est ainsi que tout projet entrepreneurial ne correspond pas forcément dans notre conception à une situation entrepreneuriale. Il est nécessaire que la décision d'agir ait été prise et qu'un certain degré d'irréversibilité du processus soit atteint notamment par le niveau d'engagement personnel et ses implications en termes de consommation de temps et d'argent. De la même façon, toute organisation entrepreneuriale «stabilisée » ne relève pas nécessairement d'une situation entrepreneuriale. L'organisation "stabilisée » (dans sa construction programmée et aménagée «chemin faisant ${ }^{10}$ ») peut très bien, à l'issue d'un certain parcours, se satisfaire de positions acquises et ne plus souhaiter la remise en cause et le changement par l'intermédiaire de la concrétisation de nouvelles occasions d'affaires. Les jeunes entités économiques ou sociales ne parviennent pas toujours à maintenir un état de situation entrepreneuriale permanent.

Dans ce contexte, centré sur le couple individu-support de la création de valeur et sur les situations entrepreneuriales, quelques questions de recherche apparaissent immédiatement :

- Comment dater et caractériser l'entrée dans une situation entrepreneuriale ?

- Comment décrire et caractériser la disparition du caractère entrepreneurial d'une situation?

10. L'expression «chemin faisant» est empruntée à Avenier (1999). 
- De quelle façon peut-on qualifier une situation entrepreneuriale ?

- Comment modéliser une situation entrepreneuriale?

- Comment l'observer et l'analyser dans l'espace et dans le temps? Avec quels instruments et outils?

- Comment intégrer l'immatérialité et l'intangibilité des supports de la création de valeur (occasions d'affaires, projet, etc.).

L'objet de recherche qui se trouve, d'après nous, au cœur de l'entrepreneuriat correspond à ces situations entrepreneuriales qui devront être décrites, expliquées et comprises dans leur dynamique et leur évolution, pour qu'à partir des connaissances d'intention scientifique produites, l'action des entrepreneurs et des structures d'appui et d'accompagnement puisse être facilitée et bonifiée. Cela n'exclut pas la définition d'autres objets de recherche situés en amont et en aval des situations entrepreneuriales et reliés à l'entrepreneuriat soit au niveau du premier cheminement, soit au niveau du second. De nombreuses questions de recherches non directement reliées à l'objet que nous avons défini présentent un intérêt majeur pour améliorer la connaissance du phénomène entrepreneurial dans son ensemble. Les travaux de Verstraete $(1997,1999)$ illustrent clairement ce point.

\section{Conclusion}

Nous avons essayé de préciser dans cette contribution notre conception de la recherche en entrepreneuriat. Pour ce faire, nous avons cherché à nous inscrire dans des tendances et à nous appuyer sur des données issues d'une revue des principaux travaux et résultats de recherche disponibles. Notre position est basée sur la conviction que pour avoir une compréhension globale du phénomène entrepreneurial, il est nécessaire d'adopter une orientation processus et d'intégrer dans un même espace d'observation toutes les facettes qui le caractérisent (Cunningham et Lischeron, 1991).

Avant de développer notre représentation, nous avons souhaité réaffirmer l'importance de la place et du rôle du chercheur en entrepreneuriat. Pour nous et dans notre discipline des sciences de gestion, il est fondamentalement un producteur de connaissances, destinées à améliorer notre compréhension de l'action des entrepreneurs.

L'objet de recherche, d'après nous, au cœur de l'entrepreneuriat est représenté par ce que nous appelons une «situation entrepreneuriale», étudiée dans sa dynamique (ensemble de configurations individu-support de création) et ses évolutions. Dans notre conception, nous pouvons parler d'entrepreneuriat lorsqu'il y 
a conjonction entre les logiques du sujet (l'individu) et de l'objet (le matériau de création de valeur). Le caractère entrepreneurial disparaît dès qu'il y a disjonction entre ces deux éléments, dès lors que ces logiques se désaccouplent. Parmi les pistes de recherche possibles, celles qui s'adressent à la nature de la relation entre ces deux logiques nous semblent prioritaires. Comment «mesurer» cette proximité ou cette distance entre une logique d'acteur et une logique de support? La relation entre conjonction et disjonction est-elle fonction du degré et du processus d'engagement? Est-elle d'essence affective? Totalement ou partiellement? Comment se construit-elle et sous l'effet de quelles forces se désagrège-t-elle ? Des réponses à ces questions dépendra vraisemblablement la solidité de cette vision.

Ce travail constitue, bien évidemment, une première étape dans un programme de recherche. Au stade actuel, nous ne cherchons pas à tester ou à vérifier un modèle théorique, mais à découvrir et à révéler ce qui est en jeu et ce qui se passe réellement dans une situation entrepreneuriale. En pensant aux prochaines étapes, nous voudrions évoquer simplement, pour terminer, quelques implications méthodologiques issues de nos réflexions.

Nous pensons tout d'abord privilégier, au moins dans un premier temps, les approches qualitatives et certaines formes de recherche-action. Nous appuyons nos convictions sur des analyses réalisées par Van de Ven (1999) dans des contextes de recherche qui nous apparaissent proches de nos propres préoccupations. Ce dernier pense, en effet, que pour acquérir une connaissance approfondie d'une activité ou d'une situation, il faut l'observer, en côtoyant ceux qui l'exercent ou ceux qui sont impliqués et réfléchir intensément sur la façon dont elle est pratiquée. Pour lui, ce type d'études est justifié par une quadruple nécessité : développer de nouvelles théories du management, confronter des propositions à la réalité du terrain (sinon il y a risque d'abstraction spéculative), élaborer des théories utiles au management, réconcilier des théories alternatives (laquelle s'applique, où et quand). Nous partageons très largement ce point de vue dans le cadre de recherche que nous avons esquissé.

D'autre part, pour définir les variables fondamentales, à l'œuvre dans une situation entrepreneuriale et pour mieux appréhender les processus de changement et de développement qui s'y déroulent, une stratégie possible de recherche semble s'imposer à nous : l'étude de cas. Elle suppose un contact approfondi avec le terrain et permet de recueillir des données d'une très grande richesse (Hlady-Rispal, 2000, 2002), utiles pour comprendre des processus relationnels, des modes de création et de fonctionnement organisationnels, des processus de décision entrepreneuriale et l'analyse des faits et des perceptions que les acteurs attachent aux actions. 


\section{Bibliographie}

Aldrich, H. (1999), Organizations Evolving, Londres, Sage Publications.

Autio, E., R. Keeley, M. Klofsten et T. Ulfstedt (1997), Entrepreneurial Intent Among Students : Testing an Intent Model in Asia, Scandinavie and USA, Wellesley, Mass., Babson College, Frontiers of Entrepreneurship Research, p. 133-147.

AVENIER, M.-J. (1999), «La complexité appelle une stratégie chemin faisant», Gestion 2000, no 5 , p. 13-34.

BAUMARD, P. (1997), Constructivisme et processus de la recherche: l'émergence d'une «posture » épistémologique chez le chercheur, Cahier de recherche 27/97, LAREGO, Université de Versailles Saint-Quentin-en-Yvelines, 18 p.

BOUCHIKHI, H. (1993), «A constructivist framework for understanding entrepreneurship performance », Organization Studies, vol. 14, no 4, p. 549-570.

Boulding, K. (1956), «General system theory: the skeleton of science», Management Science, vol. 2, p. 197-208.

BRAZEAL, D.V. et T.T. HERBERT (1999), « The genesis of entrepreneurship », Entrepreneurship Theory and Practice, vol. 23, $\mathrm{n}^{\circ}$ 1, p. 29-45.

BRUYAT, C. (1993), Création d'entreprise : contributions épistémologiques et modélisation, Thèse de doctorat en sciences de gestion, Université Pierre-Mendès-France de Grenoble, $431 \mathrm{p}$.

BRUYAT, C. (1994), «Contributions épistémologiques au domaine de l'entrepreneuriat », Revue française de gestion, $\mathrm{n}^{\circ}$ 101, p. 113-125.

BRUYAT, C. et P.-A. JULIEN (2001), «Defining the field of research in entrepreneurship », Journal of Business Venturing, vol. 16, $\mathrm{n}^{\circ}$ 2, p. 165-180.

BULL, Y. et G.E. WILLARD (1993), «Towards a theory of entrepreneurship », Journal of Business Venturing, vol. 8, n 3, p. 183-195.

BYgRaVE, W.D. et C.W. Hofer (1991), «Theorizing about entrepreneurship», Entrepreneurship Theory and Practice, vol. 15, $\mathrm{n}^{\circ}$ 1, p. 13-22.

Conti, S., E.J. MALECKI et P. Oinas (1995), The Industrial Enterprise and Its Environment: Spatial Perspectives, Aldershot, Avebury.

Cunningham, J.B. et J. Lischeron (1991), «Defining entrepreneurship», Journal of Small Business Management, vol. 29, $\mathrm{n}^{\circ}$ 1, p. 45-61.

DAvidSson, P., M.B. LOW et M. WRIGHT (2001), «Editor's introduction: Low and MacMillan ten years on: achievements and future directions for entrepreneurship research », Entrepreneurship Theory and Practice, vol. 25, n 4, p. 5-15.

DE LA VILLE, V.I. (1996), Apprentissages collectifs et structuration de la stratégie dans la jeune entreprise de haute technologie, Thèse de doctorat en sciences de gestion, Université de Lyon III.

DERY, R. et J.M. TOULOUSE (1994), «La structuration du champ de l'entrepreneurship : le cas du Journal of Business Venturing », Cahier de recherche, HEC Montréal. 
FAyOlle, A. (1996), Contribution à l'étude des comportements entrepreneuriaux des ingénieurs français, Thèse de doctorat en sciences de gestion, Université JeanMoulin de Lyon, 575 p.

FAYOLLE, A. (1999), L'enseignement de l'entrepreneuriat dans les universités françaises : analyse de l'existant et les propositions pour en faciliter le développement, Rapport d'étude rédigé à la demande de la Direction de la technologie du ministère de l'Éducation nationale, de la Recherche et de la Technologie, 101 p.

FAYOLLE, A. (2000), «L'enseignement de l'entrepreneuriat dans le système éducatif supérieur français : un regard sur la situation actuelle », Gestion 2000, n 3, p. 77-95.

FILION, L.J. (1997), «Le champ de l'entrepreneuriat : historique, évolution, tendances », Cahier de recherche $n^{\circ}$ 97.01, HEC Montréal, 36 p.

GARTNER, W.B. (1985), «A framework for describing the phenomenon of new venture creation », Academy of Management Review, vol. 10, p. 696-706.

GARTNER, W.B. (1988), «Who is an entrepreneur? Is the wrong question? », American Journal of Small Business, vol. 12, no 4, printemps, p. 11-31.

GARTNER, W.B. (1989), «Some suggestions for research on entrepreneurial traits and entrepreneurship », Entrepreneurship Theory and Practice, vol. 14, n 1, p. 27-38.

GARTNER, W.B. (1990), «What are we talking about when we talk about entrepreneurship ?», Journal of Business Venturing, vol. 5, n ${ }^{\circ}$ 1, p. 15-28.

GARTNER, W.B. (1993), «Words lead to deeds: towards an organizational emergence vocabulary », Journal of Business Venturing, vol. 8, nº 3, p. 231-239.

HeRnANDEZ, E.M. (2001), L'entrepreneuriat. Approche théorique, Paris, L'Harmattan, $270 \mathrm{p}$.

HLADY-RISPAL, M. (2000), «Une stratégie de recherche en gestion: l'étude de cas », Revue française de gestion, $\mathrm{n}^{\circ} 127, \mathrm{p} .61-79$.

HLADy-Rispal, M. (2002), La méthode des cas. Applications la recherche en gestion, Bruxelles, De Boeck Université.

Johannisson, B., C. KARLSSOn et L. Westin (1994), Patterns of a Network Economy, Londres, Springer Verlag.

JOHANNISSON, B. et H. LANDSTRÖM (1999), «Furnishing a research field», dans B. Johannisson et H. Landström (dir.), Images of Entrepreneurship and Small Business-Emergent Swedish Contributions to Academic Research, Stockholm, SIRE, p. 1-34.

KOLVEREID, L. (1996), «Organizational employment versus self-employment : reasons for career choice intentions », Entrepreneurship Theory and Practice, vol. 21, $\mathrm{n}^{\mathrm{o}} 1$, p. 23-31.

KRUEGER, N.F. et A.L. CARSRUD (1993), «Entrepreneurial intentions : applying the theory of planned behaviour », Entrepreneurship and Regional Development, vol. 5, $\mathrm{n}^{\circ} 4$, p. 315-330.

Revue internationale P.M.E., vol. 17, $\mathrm{n}^{\mathrm{o}}$ 1, 2004 
LANDSTRÖM, H. (1998), «The roots of entrepreneurship research : the intellectual development of a research field», Communication de la conférence RENT XII (Research in Entrepreneurship and Small Business), Lyon, 26-27 novembre, 18 p.

Le Moigne, J.L. (1990), La modélisation des systèmes complexes, Paris, Dunod, 178 p.

MARChESNAY, M. et P.-A. JULIEN (1989), «Small business as space of transaction», Entrepreneurship and Regional Development, vol. 2, n 3 , p. 267-277.

MARION, S. (1999), L'évaluation de projets de création d'entreprises dans le contexte d'une intervention financière, Thèse de doctorat en sciences de gestion, Université de Lyon III.

MoRIN, E. (1989), « Diriger dans la complexité », Présentation au colloque du 9 mars 1989, «Entreprise et progrès », p. 15.

Paturel, R. et H. SAVALl (1999), «Recherche en management stratégique ou management stratégique de la recherche en stratégie ?», Communication au colloque de l'AIMS, Paris, mai, 22 p.

SAmmut, S. (1995), Contribution à la compréhension du processus de démarrage en petite entreprise, Thèse de doctorat en sciences de gestion, Université de Montpellier I.

SARASVATHY, S.D. (2000), «Seminar on research perspectives in entrepreneurship », Journal of Business Venturing, vol. 15, no 1, p. 1-57.

Shane, S. et S. VenKataraman (2000), «The promise of entrepreneurship as a field of research », Academy of Management Review, vol. 25, n 1, p. 217-226.

SHARMA, P. et J.J. CHRISMAN (1999), «Toward a reconciliation of the definitional issues in the field of corporate entrepreneurship », Entrepreneurship Theory and Practice, vol. $23, \mathrm{n}^{\mathrm{0}} 3$, p. 11-28.

STEVENSON, H.H. et J.C. JARILLO (1990), «A paradigm of entrepreneurship : entrepreneurial management », Strategic Management Journal, vol. 11, n 1, p. 17-27.

Thornton, P.H. (1999), «The sociology of entrepreneurship», Annual Review of Sociology, vol. 25, p. 19-46.

TKaCheV, A. et L. Kolvereid (1999), «Self-employment intentions among Russian students », Entrepreneurship and Regional Development, vol. 11, no 3, p. 269-280.

TORNIKOSKI, E. (1999), Entrepreneurship through constructivist lenses: visionary entrepreneurship process - a conceptual development, Licentiate Thesis in Management and Organization, University of Vaasa, $167 \mathrm{p}$.

VAN DE Ven, A. (1999), «Interview d'Andrew Van de Ven», Revue française de gestion, $\mathrm{n}^{\circ} 125$, p. $58-63$.

VAn Der Veen, M. et I. WAKkeE (2002), The Entrepreneurial Process : An Overview, Proceedings, First European Summer University on Entrepreneurship Research, 19-22 septembre, Valence, France.

VENKATARAMAN, S. (1997), «The distinctive domain of entrepreneurship research », dans J.A. Katz (dir.), Advances in Entrepreneurship, Firm Emergence and Growth, vol. 3, Greenwich, Conn., JAI Press. 
VERSTRAETE, T. (1997), Modélisation de l'organisation initiée par un créateur s'inscrivant dans une logique d'entrepreneuriat persistant. Les dimensions cognitive, psychologique et struturale de l'organisation entrepreneuriale, Thèse de doctorat en sciences de gestion, Université de Lille.

Verstraete, T. (1999), Entrepreneuriat. Connaître l'entrepreneur, comprendre ses actes, Paris, L'Harmattan, 207 p.

VESPER, K. (1989), «When's the big idea », Frontiers of Entrepreneurship Research, Wellesley, Mass., Babson College, p. 334-343.

WACHEUX, F. (1996), Méthodes qualitatives et recherche en gestion, Paris, Economica, $290 \mathrm{p}$.

Wiklund, J., J. DAHLQViST et P.A. HAVNES (2001), « Entrepreneurship as new business activity: empirical evidence from young firms », Frontiers of Entrepreneurship Research, Wellesley, Mass., Babson College.

Woo, C.Y., A.C. CoOper, C. Nicholls-DiXon et W.C. DunKelberG (1990), « Adaptation by start-up firms », Frontiers of Entrepreneurship Research, Wellesley, Mass., Babson College, p. 132-143.

WORTMAN, M. Jr. (1987), «Entrepreneurship: an integrating typology and evaluation of the empirical research in the field», Journal of Management, vol. 13, n ${ }^{\circ} 3$, p. 259-279.

Revue internationale P.M.E., vol. 17, $\mathrm{n}^{\mathrm{o}}$ 1, 2004 